\title{
SCHRÖDINGER EQUATION FOR A PARTICLE ON A CURVED SPACE AND SUPERINTEGRABILITY
}

\section{PAUL BRACKEN}

\section{Communicated by Vasil V. Tsanov}

\begin{abstract}
A formulation of quantum mechanics on spaces of constant curvature is studied by quantizing the Noether momenta and using these to form the quantum Hamiltonian. This approach gives the opportunity of studying a superintegrable quantum system. It is shown there are three different ways of obtaining a Hilbert space of common eigenstates. Three different orthogonal coordinate systems are determined, one for each case. It is shown how the Schrödinger equation can be rendered separable in each of the cases.
\end{abstract}

MSC: 81S10, 51P05, 35P05

Keywords: curvature, vector field, Hamiltonian, quantization, metric, canonical transformation

\section{Introduction}

The study of a quantum free particle in Euclidean space leads to the straightforward conclusion that the solutions are plane-wave states that are in fact eigenfunctions of the linear momentum operator. Plane waves are therefore simultaneous eigenfunctions of energy and linear momentum. As soon as the problem is thought of in a space with curvature, the analysis becomes much more complicated $[11,14,15]$. First of all, the canonical momenta do not in general coincide with the Noether momenta. Secondly, the Noether momenta do not Poisson commute classically, so the corresponding self-adjoint quantum operators do not commute. A planewave is more of a Euclidean concept, and its meaning needs to be clarified in a curved space. The approach taken here is mainly suited for discussing questions which arise in applications of nonrelativistic quantum mechanics. For example, a two-dimensional application of quantum mechanics arises in condensed matter physics. This is the existence of Landau levels for the motion of a charged particle under perpendicular magnetic fields. This problem and its application to the quantum Hall effect has been studied before in the case of non-Euclidean geometries. 
There has been a renewed interest in submanifold quantum mechanics, especially with regard to applications of quantum motion on a submanifold under the generalized Dirac theory of second-class constraints [10,16]. In [12], an algebraic definition of submanifold quantum mechanics is given, which is free from any approximation theories. The essentials of submanifold quantum mechanics is given from an algebraic point of view. An extensive discussion of the area of quantization on curved manifolds appears in [13]. Here one particular curvature dependent approach will be introduced and a quantization is utilized which results in a separable Schrödinger equation. It is the objective to look at the mathematical structure of the separability of this equation. This type of model has been considered previously [4,7], and after some classical aspects of the model are presented [1,9], the superintegrability of the model not touched on in [2,3] is investigated in detail.

Curvature is introduced as a constant parameter in the metric to describe the space in such a way that, for different sign choices, one of the following three spaces $\left(S_{\kappa}^{2}, \mathbb{R}^{2}, H_{\kappa}^{2}\right)$ of constant curvature $\kappa$ is obtained. Spherical dynamics results when $\kappa>0$ and hyperbolic dynamics holds in the case $\kappa<0$, so the case $\kappa=0$ is Euclidean space. These spaces can be collectively referred to as the manifold $M_{\kappa}^{2}$. In fact, this approach can be initiated in a way that makes calculation practical by defining the metric to have a certain structure. The main intent is to investigate closely a method under which the quantization of the system can be done in general and then to take a close look at the separability of the resulting Schrödinger equation. It is interesting to find in this approach that there are three different sets of compatible observables, and therefore, three different ways of obtaining a Hilbert space of common eigenstates. Three different orthogonal coordinate systems will be determined explicitly in each of the three cases. The separation of the Schrödinger equation is carried out in detail for each one. It should be quite clear at each step what is occurring with regard to the physics of the problem. In fact, it will be shown that multiple separability can be demonstrated and the existence of multiple separability is a property which is directly related with what has come to be known as the superintegrability of a system.

\section{Metric and Noether Symmetries}

The manifold $M_{\kappa}^{2}$ is given the following metric

$$
g=\frac{1}{1-\kappa r^{2}}\left(\left(1-\kappa y^{2}\right) \mathrm{d} x \otimes \mathrm{d} x+\left(1-\kappa x^{2}\right) \mathrm{d} y \otimes \mathrm{d} y+\kappa x y \mathrm{~d} x \otimes \mathrm{d} y+\kappa x y \mathrm{~d} y \otimes \mathrm{d} x\right) .
$$


The tensor $g$ in (1) can also be put in the form

$$
g=\frac{1}{1-\kappa r^{2}}\left(\mathrm{~d} x \otimes \mathrm{d} x+\mathrm{d} y \otimes \mathrm{d} y-\kappa(x \mathrm{~d} y-y \mathrm{~d} x)^{2}\right), \quad r^{2}=x^{2}+y^{2} .
$$

Define a vector field $X$ on $M_{\kappa}^{2}$ in terms of functions $f$ and $g$ as

$$
X=f(x, y) \frac{\partial}{\partial x}+h(x, y) \frac{\partial}{\partial y} .
$$

The unknown functions $f$ and $h$ will be determined such that the Lie derivative of metric $g$ with respect to $X$ vanishes

$$
\mathcal{L}_{X} g=0
$$

The like terms in (4) are collected together and (4) will be satisfied provided that each of the tensor coefficients vanishes. This yields a system of three independent partial differential equations in terms of the functions in $X$.

Proposition 1. The Lie derivative of metric (1) vanishes if and only if the following system of coupled partial differential equations for $f$ and $h$ is satisfied

$$
\begin{gathered}
\left(f \frac{\partial}{\partial x}+h \frac{\partial}{\partial y}\right)\left(\frac{1-\kappa y^{2}}{1-\kappa r^{2}}\right)+2 \frac{1-\kappa y^{2}}{1-\kappa r^{2}} \frac{\partial f}{\partial x}+2 \frac{\kappa x y}{1-\kappa r^{2}} \frac{\partial h}{\partial x}=0 \\
\frac{1-\kappa y^{2}}{1-\kappa r^{2}} \frac{\partial f}{\partial y}+\frac{1-\kappa x^{2}}{1-\kappa r^{2}} \frac{\partial h}{\partial x}+\frac{\kappa x y}{1-\kappa r^{2}}\left(\frac{\partial f}{\partial x}+\frac{\partial h}{\partial y}\right)+\left(f \frac{\partial}{\partial x}+h \frac{\partial}{\partial y}\right) \frac{\kappa x y}{1-\kappa r^{2}}=0 \\
\left(f \frac{\partial}{\partial x}+h \frac{\partial}{\partial y}\right) \frac{1-\kappa x^{2}}{1-\kappa r^{2}}+2 \frac{1-\kappa x^{2}}{1-\kappa r^{2}} \frac{\partial h}{\partial y}+2 \frac{\kappa x y}{1-\kappa r^{2}} \frac{\partial f}{\partial y}=0 .
\end{gathered}
$$

Moreover, there exists a general solution to (5) for the functions $f$ and $h$ which is

$$
f(x, y)=C_{2} y+C_{1} \sqrt{1-\kappa r^{2}}, \quad h(x, y)=-C_{2} x+C_{3} \sqrt{1-\kappa r^{2}} .
$$

A single solution will suffice, so the arbitrary constants can be selected to be $\left(C_{1}, C_{2}, C_{3}\right)=(-1,0,0),(0,0,1),(0,-1,0)$. A particular solution for the three independent vector fields $X_{j}$ is then given by

$$
X_{1}(\kappa)=-\sqrt{1-\kappa r^{2}} \frac{\partial}{\partial x}, \quad X_{2}(\kappa)=\sqrt{1-\kappa r^{2}} \frac{\partial}{\partial y}, \quad X_{j}=x \frac{\partial}{\partial y}-y \frac{\partial}{\partial x}
$$

By means of long calculation aided by the use of symbolic manipulation [8], it can be verified that the commutator brackets of the vector fields in (6) satisfy

$$
\left[X_{1}(\kappa), X_{2}(\kappa)\right]=\kappa X_{J}, \quad\left[X_{1}(\kappa), X_{J}\right]=-X_{2}(\kappa), \quad\left[X_{2}(\kappa), X_{J}\right]=X_{1}(\kappa)
$$


The Lie algebra corresponding to the brackets closes according to the sign of the curvature $\kappa$ as the Lie algebra of isometries of spherical, Euclidean and hyperbolic spaces.

From the physical point of view, the geodesic motion on $M_{\kappa}^{2}$ is determined by a Lagrangian $L$ which consists of a $\kappa$-dependent kinetic term $T(\kappa)$ without a potential term

$$
L(\kappa)=\frac{1}{2} \frac{1}{1-\kappa r^{2}}\left(v_{x}^{2}+v_{y}^{2}-\kappa\left(x v_{y}-y v_{x}\right)^{2}\right)
$$

and the parameter $\kappa$ may take on both positive and negative values. In the spherical case, when $\kappa>0$, the function and associated dynamics has a singularity at $1-$ $\kappa r^{2}=0$. In this event, the study of the dynamics is restricted to $r^{2}<1 / \kappa$ so that the kinetic energy function is positive definite. This free-particle Lagrangian is invariant under the action of the three vector fields (6) in the sense that if $X_{j}^{t}$, $j=1,2,3$ denotes the natural lift to the tangent bundle or phase space of vector field $X_{j}$, the Lie derivative of $L(\kappa)$ with respect to $X_{j}^{t}$ will vanish.

A Lagrangian for the problem is naturally defined by means of the metric (1)

$$
L(\kappa)=\frac{1}{2} g_{i j} v^{i} v^{j} .
$$

The momenta $p_{x}, p_{y}$ are obtained by differentiation of the Lagrangian

$$
p_{x}=\frac{1}{1-\kappa r^{2}}\left(v_{x}+\kappa\left(x v_{y}-y v_{x}\right) y\right), \quad p_{y}=\frac{1}{1-\kappa r^{2}}\left(v_{y}-\kappa\left(x v_{y}-y v_{x}\right) x\right) .
$$

Solving for $v_{x}, v_{y}$ from (9), we get

$$
v_{x}=\left(1-\kappa x^{2}\right) p_{x}-\kappa x y p_{y}, \quad v_{y}=\left(1-\kappa y^{2}\right) p_{y}-\kappa x y p_{x} .
$$

The Legendre transformation leads to the following expression for the $\kappa$-dependent Hamiltonian based on the metric (1)

$$
H=\frac{1}{2}\left(p_{x}^{2}+p_{y}^{2}-\kappa\left(x p_{x}+y p_{y}\right)^{2}\right)
$$

and the Noether momenta take the form

$$
P_{1}(\kappa)=\sqrt{1-\kappa r^{2}} p_{x}, \quad P_{2}(\kappa)=\sqrt{1-\kappa r^{2}} p_{y}, \quad J=x p_{y}-y p_{x} .
$$

The quantities in (12) satisfy the following Poisson brackets

$$
\left\{P_{1}(\kappa), P_{2}(\kappa)\right\}=\kappa J, \quad\left\{P_{1}(\kappa), J\right\}=-P_{2}(\kappa), \quad\left\{P_{2}(\kappa), J\right\}=P_{1}(\kappa) .
$$

Solving (12) for $p_{x}$ and $p_{y}$, the Hamiltonian (11) can be transformed into the Noether momenta (12) as follows

$$
H(\kappa)=\frac{1}{2}\left(P_{1}^{2}+P_{2}^{2}+\kappa J^{2}\right) .
$$


In fact, $H(\kappa)$ is just the Casimir for the Poisson algebra given by (13).

There is a measure on $\mathbb{R}^{2}$ that is invariant under the action of the set of vector fields $\left\{X_{1}, X_{2}, J\right\}$ and up to a constant factor, it is given by

$$
\mathrm{d} \mu_{\kappa}=\frac{1}{\sqrt{1-\kappa r^{2}}} \mathrm{~d} x \wedge \mathrm{d} y
$$

\section{Quantization of the Hamiltonian and Schrödinger Equation}

The operator Hamiltonian is now defined so that it is self-adjoint in the Hilbert space $L_{\kappa}^{2}\left(\mathrm{~d} \mu_{\kappa}\right)$. To carry this out, consider the operators $\hat{P}_{1}, \hat{P}_{2}$ which represent the quantum version of the Noether momenta $P_{1}, P_{2}$ in (12) that are self-adjoint in the space $L^{2}\left(\mathrm{~d} \mu_{\kappa}\right)$. They are given by

$$
\hat{P}_{1}=-\mathrm{i} \hbar \sqrt{1-\kappa r^{2}} \frac{\partial}{\partial x}, \quad \hat{P}_{2}=-\mathrm{i} \hbar \sqrt{1-\kappa r^{2}} \frac{\partial}{\partial y} .
$$

Substituting operators (16) into Hamiltonian (14), the quantum Hamiltonian $\hat{H}(\kappa)$ is given as

$$
\begin{aligned}
\hat{H}(\kappa)= & -\frac{\hbar^{2}}{2 m}\left(\left(1-\kappa r^{2}\right) \frac{\partial^{2}}{\partial x^{2}}-\kappa x \frac{\partial}{\partial x}\right)-\frac{\hbar^{2}}{2 m}\left(\left(1-\kappa r^{2}\right) \frac{\partial^{2}}{\partial y^{2}}-\kappa y \frac{\partial}{\partial y}\right) \\
& -\kappa \frac{\hbar^{2}}{2 m}\left(x^{2} \frac{\partial^{2}}{\partial y^{2}}+y^{2} \frac{\partial^{2}}{\partial x^{2}}-2 x y \frac{\partial^{2}}{\partial x \partial y}-x \frac{\partial}{\partial x}-y \frac{\partial}{\partial y}\right) .
\end{aligned}
$$

Clearly, this Hamiltonian admits the following decomposition

$$
\hat{H}(\kappa)=\hat{H}_{1}+\hat{H}_{2}+\kappa \hat{J}^{2} .
$$

and since the separability of this problem will be studied, the components of (18) will be given explicitly

$$
\begin{gathered}
\hat{H}(\kappa)=-\frac{\hbar^{2}}{2 m}\left(\left(1-\kappa r^{2}\right) \frac{\partial^{2}}{\partial x^{2}}-\kappa x \frac{\partial}{\partial x}\right) \\
\hat{H}_{2}(\kappa)=-\frac{\hbar^{2}}{2 m}\left(\left(1-\kappa r^{2}\right) \frac{\partial^{2}}{\partial y^{2}}-\kappa y \frac{\partial}{\partial y}\right) \\
\hat{J}^{2}=-\frac{\hbar^{2}}{2 m}\left(x^{2} \frac{\partial^{2}}{\partial y^{2}}+y^{2} \frac{\partial^{2}}{\partial x^{2}}-2 x y \frac{\partial^{2}}{\partial x \partial y}-x \frac{\partial}{\partial x}-y \frac{\partial}{\partial y}\right) .
\end{gathered}
$$

By means of computer calculation, it can be shown that the total Hamiltonian $\hat{H}$ in (18) commutes with all of the related operators in (19)-(21) for any $\kappa$

$$
\left[\hat{H}(\kappa), \hat{H}_{1}(\kappa)\right]=0, \quad\left[\hat{H}(\kappa), \hat{H}_{2}(\kappa)\right]=0, \quad\left[\hat{H}(\kappa), \hat{J}^{2}\right]=0 .
$$


The vanishing of these brackets means that the $\kappa$-dependent Hamiltonian $\hat{H}(\kappa)$ describes a superintegrable quantum system. This property was well known in the Euclidean case, and appears in a slightly different form in the case $M_{\kappa}^{2}$ because of the $\hat{J}^{2}$ term.

\section{Schrödinger Equation and Its Separability}

These results offer the possibility of studying separability of the eigenvalue problems which are relevant to the operators (19)-(21). The Schrödinger equation corresponding to (18) is

$$
\hat{H} \Psi=E \Psi .
$$

By means of the same type of calculations that produced the brackets in (8), the operators in (19)-(21) are found to satisfy the following brackets

$$
\left[\hat{H}_{1}, \hat{H}_{2}+\kappa \hat{J}^{2}\right]=0, \quad\left[\hat{H}_{1}+\kappa \hat{J}^{2}, \hat{H}_{2}\right]=0, \quad\left[\hat{H}_{1}+\hat{H}_{2}, \hat{J}^{2}\right]=0 .
$$

This justifies the earlier claim that there are three different sets of compatible observables, hence three different ways of obtaining a Hilbert space of common eigenstates. These three descriptions are a direct consequence of the $\kappa \hat{J}^{2}$ term in the kinetic part of the Hamiltonian. The metric $g$ is not diagonal in the $(x, y)$ coordinates and Schrödinger equation (23) is not separable in these due to this $\kappa$-term. However, the classical Hamilton-Jacobi equation, which is

$$
\left(\frac{\partial S}{\partial x}\right)^{2}+\left(\frac{\partial S}{\partial y}\right)^{2}-\kappa\left(x \frac{\partial S}{\partial x}+y \frac{\partial S}{\partial y}\right)^{2}=0
$$

and the quantum Schrödinger equation are separable in three different orthogonal coordinate systems. It is the purpose here to show how these coordinate systems can be determined explicitly. and to give a well-defined procedure for diagonalizing these operators. Before looking at each of the three cases, let us obtain a general transformation by first considering the pair of differentiable functions

$$
x=s(r, \varphi), \quad y=t(r, \varphi) .
$$

By means of the chain rule, the basis $\left\{\partial_{r}, \partial_{\varphi}\right\}$ can be expressed in terms of the set $\left\{\partial_{x}, \partial_{y}\right\}$, and by solving, $\left\{\partial_{x}, \partial_{y}\right\}$ can be expressed in terms of $\left\{\partial_{r}, \partial_{\varphi}\right\}$ as well

$$
\frac{\partial}{\partial x}=\frac{1}{W}\left(\frac{\partial t}{\partial r} \frac{\partial}{\partial \varphi}-\frac{\partial t}{\partial \varphi} \frac{\partial}{\partial r}\right), \quad \frac{\partial}{\partial y}=\frac{1}{W}\left(\frac{\partial s}{\partial \varphi} \frac{\partial}{\partial r}-\frac{\partial s}{\partial r} \frac{\partial}{\partial \varphi}\right)
$$

where

$$
W=\frac{\partial s}{\partial \varphi} \frac{\partial t}{\partial r}-\frac{\partial s}{\partial r} \frac{\partial t}{\partial \varphi}
$$


Let us go through each of the three cases presented in (24) in detail.

Case 1. The first problem to be studied is $\left[\hat{H}_{1}, \hat{H}_{2}+\kappa \hat{J}^{2}\right]=0$. The objective in specifying the functions in (26) more explicitly is to eliminate from $\hat{H}_{1}$ and $\hat{H}_{2}+\kappa \hat{J}^{2}$ all mixed partial derivatives in transforming between the $(x, y)$ and $(r, \varphi)$ systems. Denote for convenience by $\tilde{H}_{2}$ and $\tilde{J}$ the operators in $x$ and $y$ but omitting the physical constants in front

$$
\tilde{H}_{2}=\left(1-\kappa r^{2}\right) \frac{\partial^{2}}{\partial y^{2}}-\kappa y \frac{\partial}{\partial y}, \quad \tilde{J}=x \frac{\partial}{\partial y}-y \frac{\partial}{\partial x}, \quad r^{2}=x^{2}+y^{2} .
$$

The operator $\tilde{H}_{2}+\kappa \tilde{J}^{2}$ is formed from the operators in (28). It is now required to obtain a transformation of coordinates (26) such that no mixed derivatives occur in the transformed operator. By a long calculation, it is found that an appropriate transformation can be obtained in the form

$$
x=s(r, \varphi)=r \cos \varphi, \quad y=t(r, \varphi)=\frac{1}{\sqrt{\kappa}} \sin \varphi .
$$

By direct calculation as well, the operator $\tilde{H}_{2}+\kappa \tilde{J}^{2}$ is transformed into

$$
\tilde{H}_{2}+\kappa \tilde{J}^{2}=\tan ^{2} \varphi\left(\left(1-\kappa r^{2}\right) \frac{\partial^{2}}{\partial r^{2}}-\kappa r \frac{\partial}{\partial r}\right)+\kappa\left(\frac{\partial^{2}}{\partial \varphi^{2}}-\tan \varphi \frac{\partial}{\partial \varphi}\right) .
$$

Since $y$ in (29) is proportional to $\sin \varphi$ and $r$ does not appear, (30) can be returned to the $y$-variable by first introducing the new variable $z_{x}$ which is related to $x$ and defined as

$$
z_{x}=r=\frac{x}{\sqrt{1-\sin ^{2} \varphi}}=\frac{x}{\sqrt{1-\kappa y^{2}}} .
$$

To go from $\varphi$ back to the $y$-variable, the following relations are required for the derivatives

$$
\frac{x}{y}=\sqrt{\kappa} r \cot \varphi, \quad \cot ^{2} \varphi=\frac{x^{2}}{\kappa r^{2} y^{2}}=\frac{1-\kappa y^{2}}{\kappa y^{2}}, \quad \sin \varphi=\sqrt{\kappa} y .
$$

Then the $\varphi$-derivatives can be expressed as follows

$$
\frac{\partial}{\partial \varphi}=\frac{\sqrt{1-\kappa y^{2}}}{\sqrt{\kappa}} \frac{\partial}{\partial y}, \quad \frac{\partial^{2}}{\partial \varphi^{2}}=\frac{1}{\kappa}\left(1-\kappa y^{2}\right) \frac{\partial^{2}}{\partial y^{2}}-y \frac{\partial}{\partial y} .
$$

Substituting these results into the operators $\tilde{H}_{1}$ and $\tilde{H}_{2}+\kappa \tilde{J}^{2}$, it is found that

$$
\begin{aligned}
c \tilde{H}_{1} & =\left(1-\kappa z_{x}^{2}\right) \frac{\partial^{2}}{\partial z_{x}^{2}}-\kappa z_{x} \frac{\partial}{\partial z_{x}} \\
\tilde{H}_{2}+\kappa \tilde{J}^{2} & =\frac{\kappa y^{2}}{1-\kappa y^{2}}\left(\left(1-\kappa z_{x}^{2}\right) \frac{\partial^{2}}{\partial z_{x}^{2}}-\kappa z_{x} \frac{\partial}{\partial z_{x}}\right)+\left(1-\kappa y^{2}\right) \frac{\partial^{2}}{\partial y^{2}}-2 \kappa y \frac{\partial}{\partial y} .
\end{aligned}
$$


Clearly, the problem has decoupled in the $\left(z_{x}, y\right)$ variables. In fact, substituting $\tilde{H}_{1}$ in (32) into the second operator, it takes the form

$$
\tilde{H}_{2}+\kappa \tilde{J}^{2}=\frac{\kappa y^{2}}{1-\kappa y^{2}} \tilde{H}_{1}+\left(\left(1-\kappa y^{2}\right) \frac{\partial^{2}}{\partial y^{2}}-2 \kappa y \frac{\partial}{\partial y}\right) .
$$

These calculations show that the $\kappa$-dependent Schrödinger equation is in fact separable in these new coordinates, and this two-dimensional problem has been decoupled into two one-dimensional problems. Since

$$
\hat{H}_{1}=-\frac{\hbar^{2}}{2 m} \tilde{H}_{1}
$$

the Schrödinger equation

$$
\hat{H}_{1} \Psi=\mathcal{E}_{1} \Psi
$$

for the first partial Hamiltonian $\hat{H}_{1}$ leads to the following equation with $z_{x}$ derivatives

$$
\left(1-\kappa z_{x}^{2}\right) \Psi_{z_{x} z_{x}}-\kappa z_{x} \Psi_{z_{x}}+\mu \Psi=0, \quad \mu=\frac{2 m}{\hbar^{2}} \mathcal{E}_{1} .
$$

Substituting (34) into (33), the Schrödinger equation for the second partial Hamiltonian leads to the $\mu$-dependent equation with $y$-derivatives

$$
-\frac{\kappa y^{2}}{1-\kappa y^{2}}(\mu \Psi)+\left(1-\kappa y^{2}\right) \Psi_{y y}-(2 \kappa y) \Psi_{y}+\nu \Psi=0, \quad \nu=\frac{2 m}{\hbar^{2}} \mathcal{E}_{2 j} .
$$

Now the function $\Psi\left(z_{x}, y\right)$ can be taken in the form

$$
\Psi\left(z_{x}, y\right)=Z\left(z_{x}\right) Y(y) .
$$

Then the pair of equations (35) and (36) separate and simplify to the following form

$$
\begin{gathered}
\left(1-\kappa z_{x}^{2}\right) Z_{z_{x} z_{x}}-\kappa z_{x} Z_{z_{x}}+\mu Z=0 \\
\left(1-\kappa y^{2}\right) Y_{y y}-2 \kappa y Y_{y}-\mu \kappa \frac{y^{2}}{1-\kappa y^{2}} Y+\nu Y=0 .
\end{gathered}
$$

Case 2. The second class is closely related to the first, and proceeds along exactly the same lines and is concerned with the observables $\left[\hat{H}_{2}, \hat{H}_{1}+\kappa \hat{J}^{2}\right]=0$. A transformation of the form (26) is given as

$$
x=s(r, \varphi)=\frac{1}{\sqrt{\kappa}} \cos \varphi, \quad y=t(r, \varphi)=r \sin \varphi .
$$

Then by direct calculation, the operator $\tilde{H}_{1}+\kappa \tilde{J}^{2}$ ends in the form

$$
\tilde{H}_{1}+\kappa \tilde{J}^{2}=\cot ^{2} \varphi\left(\left(1-\kappa r^{2}\right) \frac{\partial^{2}}{\partial r^{2}}-\kappa r \frac{\partial}{\partial r}\right)+\kappa\left(\frac{\partial^{2}}{\partial \varphi^{2}}+\cot \varphi \frac{\partial}{\partial \varphi}\right) .
$$


Since $x$ is proportional to $\cos \varphi,(40)$ can be written in terms of the variable $x$ by introducing the new variable $z_{y}$ defined by

$$
z_{y}=r=\frac{y}{\sqrt{1-\cos ^{2} \varphi}}=\frac{y}{\sqrt{1-\kappa x^{2}}} .
$$

Then it follows that

$$
\frac{y}{x}=\sqrt{\kappa} r \tan \varphi, \quad \tan ^{2} \varphi=\frac{y^{2}}{\kappa x^{2} r^{2}}=\frac{1-\kappa x^{2}}{\kappa x}, \quad \cos \varphi=\sqrt{\kappa} x .
$$

The derivatives in $\varphi$ transform as

$$
\frac{\partial}{\partial \varphi}=-\frac{\sqrt{1-\kappa x^{2}}}{\sqrt{\kappa}} \frac{\partial}{\partial x}, \quad \frac{\partial^{2}}{\partial \varphi^{2}}=\frac{1}{\kappa}\left(1-\kappa x^{2}\right) \frac{\partial^{2}}{\partial x^{2}}-x \frac{\partial}{\partial x} .
$$

Substituting these results into (40), it is found that

$$
\tilde{H}_{1}+\kappa \tilde{J}^{2}=\frac{\kappa x^{2}}{1-\kappa x^{2}}\left(\left(1-\kappa z_{y}^{2}\right) \frac{\partial^{2}}{\partial z_{y}^{2}}-\kappa z_{y} \frac{\partial}{\partial z_{y}}\right)+\left(1-\kappa x^{2}\right) \frac{\partial^{2}}{\partial x^{2}}-2 \kappa x \frac{\partial}{\partial x} .
$$

Then the Hamiltonian $\hat{H}_{2}$ is given by

$$
\hat{H}_{2}=-\frac{\hbar^{2}}{2 m} \tilde{H}_{2}, \quad \tilde{H}_{2}=\left(1-\kappa z_{y}^{2}\right) \frac{\partial^{2}}{\partial z_{y}^{2}}-\kappa z_{y} \frac{\partial}{\partial z_{y}} .
$$

The Schrödinger equation decouples in the new $\left(x, z_{y}\right)$ variables. The operator $\tilde{H}_{1}+\kappa \tilde{J}^{2}$ takes the following form

$$
\tilde{H}_{1}+\kappa \tilde{J}^{2}=\frac{\kappa x^{2}}{1-\kappa x^{2}} \tilde{H}_{2}+\left(\left(1-\kappa x^{2}\right) \frac{\partial^{2}}{\partial x^{2}}-2 \kappa x \frac{\partial}{\partial x}\right) .
$$

The $\kappa$-dependent Schrödinger equation is in fact separable in these new coordinates, and again, the two-dimensional problem has been decoupled into two onedimensional systems.

The equation $\hat{H}_{2} \Psi=\mathcal{E}_{2} \Psi$ for the partial Hamiltonian $\hat{H}_{2}$ leads to the following equation with derivatives in the variable $z_{y}$

$$
\left(1-\kappa z_{y}^{2}\right) \Psi_{z_{y} z_{y}}-\kappa z_{y} \Psi_{z_{y}}+\mu \Psi=0, \quad \mu=\frac{2 m}{\hbar^{2}} \mathcal{E}_{2}
$$

The Schrödinger equation $\left(\hat{H}_{1}+\kappa \hat{J}^{2}\right) \Psi=\mathcal{E}_{1 j} \Psi$ for the first partial Hamiltonian leads to the following $\mu$-dependent equation in the $x$ derivatives

$$
-\frac{\kappa x^{2}}{1-\kappa x^{2}}(\mu \Psi)+\left(\left(1-\kappa x^{2}\right) \Psi_{x x}-2 \kappa x \Psi_{x}\right)+\nu \Psi=0, \quad \nu=\frac{2 m}{\hbar^{2}} \mathcal{E}_{1 j} .
$$


Suppose the wave function is written in separable form as

$$
\Psi\left(x, z_{y}\right)=X(x) Z\left(z_{y}\right)
$$

the pair of equations in $Z$ and $X$ simplify to the following forms

$$
\begin{gathered}
\left(1-\kappa z_{y}^{2}\right) Z_{z_{y} z_{y}}-\kappa z_{y} Z_{z_{y}}+\mu Z=0 \\
\left(1-\kappa x^{2}\right) X_{x x}-2 \kappa x X_{x}-\kappa \mu\left(\frac{x^{2}}{1-\kappa x^{2}}\right) X+\nu X=0 .
\end{gathered}
$$

Note that the $(Z, Y)$ equations in Case 1 are identical to the $(Z, X)$ equations in Case 2 after a minor relabelling.

Case 3. The third case can also be examined by means of the same type of transformation. In fact, usual polar coordinates in (26) will suffice

$$
x=r \cos \varphi, \quad y=r \sin \varphi .
$$

Since the operators $\hat{H}_{1}$ and $\hat{H}_{2}$ can be expressed in the $(x, y)$ system as

$$
\tilde{H}_{1}=\left(1-\kappa r^{2}\right) \frac{\partial^{2}}{\partial x^{2}}-\kappa x \frac{\partial}{\partial x}, \quad \tilde{H}_{2}=\left(1-\kappa r^{2}\right) \frac{\partial^{2}}{\partial y^{2}}-\kappa y \frac{\partial}{\partial y} .
$$

Transforming the sum $\tilde{H}_{1}+\tilde{H}_{2}$ into polar coordinates, it is found that

$$
\tilde{H}_{1}+\tilde{H}_{2}=\left(1-\kappa r^{2}\right)\left(\frac{\partial^{2}}{\partial r^{2}}+\frac{1}{r} \frac{\partial}{\partial r}+\frac{1}{r^{2}} \frac{\partial^{2}}{\partial \varphi^{2}}\right) .
$$

It follows then that this system is described by the following pair of equations

$$
\left(\hat{H}_{1}+\hat{H}_{2}\right) \Psi=\mu_{12} \Psi, \quad \hat{J}^{2} \Psi=\nu_{j} \Psi .
$$

where $\mu_{12}$ and $\nu_{j}$ are defined to be

$$
\mu_{12}=-\frac{2 m}{\hbar} \mathcal{E}_{12}, \quad \nu_{j}=-\frac{2 m}{\hbar^{2}} \mathcal{E}_{j} .
$$

In terms of differential operators, (53) assumes the form

$$
\left(1-\kappa r^{2}\right)\left(\frac{\partial^{2}}{\partial r^{2}}+\frac{1}{r} \frac{\partial}{\partial r}+\frac{1}{r^{2}} \frac{\partial^{2}}{\partial \varphi^{2}}\right) \Psi=\mu_{12} \Psi, \quad \frac{\partial^{2} \Psi}{\partial \varphi^{2}}=\nu_{j} \Psi .
$$

An equation with only $r$ derivatives can be obtained by putting the second equation in (55) into the first. If it is then assumed that $\Psi(r, \varphi)$ separates into the form

$$
\Psi(r, \varphi)=R(r) U(\varphi)
$$

then the pair of equations in (55) takes the form

$$
\begin{aligned}
\left(1-\kappa r^{2}\right)\left(\frac{\partial^{2}}{\partial r^{2}}+\frac{1}{r} \frac{\partial}{\partial r}\right) R(r)+\frac{1}{r^{2}}\left(1-\kappa r^{2}\right) \nu_{j} R(r) & =\mu_{12} R(r) \\
\frac{\partial^{2} A(\varphi)}{\partial \varphi^{2}} & =\nu_{j} A(\varphi) .
\end{aligned}
$$




\section{Resolution of Equations}

Considerable effort could now be expended in studying the differential equations which have resulted from this analysis, however, this will not be carried out here. Since the equations obtained in the first two cases are identical up to a change of notation, only an overview of what can be done in these two cases when $\kappa$ is negative will be presented.

First, consider the equation for $Z$ in the hyperbolic region. Suppose a negative value for $\kappa$ is assumed so that $\kappa=-|\kappa|<0$. Then the equation for $Z$ takes the form

$$
\left(1+|\kappa| z_{x}^{2}\right) Z_{z_{x} z_{x}}+|\kappa| z_{x} Z_{z_{x}}+\mu Z=0 .
$$

Since $z_{x} \rightarrow x$ as $\kappa \rightarrow 0$, a form of $Z$ that goes into a plane wave may be assumed which has the form

$$
Z=\mathrm{e}^{\mathrm{i} u\left(z_{x}\right)}
$$

where $u\left(z_{x}\right)$ is an unknown function to be determined. Substituting (59) into (58), the following equation is obtained

$$
\left(1+|\kappa| z_{x}^{2}\right) u_{z_{x} z_{x}}+\mathrm{i}\left(1+|\kappa| z_{x}^{2}\right) u_{z_{x}}^{2}+\kappa z_{x} u_{z_{x}}-\mathrm{i} \mu=0 .
$$

There exists a solution to this equation of the form

$$
u\left(z_{x}\right)=\sqrt{\frac{\mu}{|\kappa|}} \operatorname{arcsinh}\left(\sqrt{|\kappa|} z_{x}\right) .
$$

This is a well-defined function for all values of the variable $z_{x}$ and there are no natural restrictions on the domain of $z_{x}$. Since $\kappa \rightarrow 0$ corresponds to the Euclidean limit, and $z_{x} \rightarrow x$, the solution (61) satisfies the appropriate Euclidean limit

$$
\lim _{\kappa \rightarrow 0} Z\left(z_{x}\right)=A \mathrm{e}^{\mathrm{i} k_{x} x}+B \mathrm{e}^{-\mathrm{i} k_{x} x} .
$$

This solution can be thought of as representing a $\kappa$-dependent hyperbolic deformation of the Euclidean plane wave solution.

The equations for $Y$ and $X$ have the same structure. Hence, it suffices to consider the $Y$ equation, which assumes the form

$$
\left(1+|\kappa| y^{2}\right) Y_{y y}+2|\kappa| y Y_{y}+\mu|\kappa| \frac{y^{2}}{1+|\kappa| y^{2}} Y+\nu Y=0
$$

A hypergeometric equation can be obtained from (62), but to do so, the following factorization must be considered

$$
Y(y)=p(y)\left(1+|\kappa| y^{2}\right)^{g / 2} .
$$


Substituting (63) into (62), this leads to the constraint $g^{2}|\kappa|+\mu=0$, hence $g$ is complex

$$
g=\mathrm{i} \sqrt{\frac{\mu}{|\kappa|}}=\mathrm{i} g_{\kappa} .
$$

This implies that a complex hypergeometric equation should result. Assuming the complex factorization

$$
Y(y)=P(y)\left(1+|\kappa| y^{2}\right)^{\mathrm{i} g_{\kappa} / 2}, \quad P(y)=p_{1}(y)+\mathrm{i} p_{2}(y)
$$

then substituting $Y(y)$ in (64) into (62), it is found that $P(y)$ satisfies the following equation

$$
\left(1+|\kappa| y^{2}\right) P_{y y}+2|\kappa|\left(1+\mathrm{i} g_{\kappa}\right) y P_{y}+\left(\nu+\mathrm{i}|\kappa| g_{\kappa}\right) P=0 .
$$

Equation (65) is a complex hypergeometric equation. Substituting the complex form for $P(y)$ in (64) into (65), it is easy to see that (65) can be expressed as a system of two coupled real equations

$$
\begin{aligned}
& \left(1+|\kappa| y^{2}\right) p_{1, y y}+2|\kappa| y p_{1, y}+\nu p_{1}=|\kappa| g_{\kappa}\left(2 y p_{2, y}+p_{2}\right) \\
& \left(1+|\kappa| y^{2}\right) p_{2, y y}+2|\kappa| y p_{2, y}+\nu p_{2}=-|\kappa| g_{\kappa}\left(2 y p_{1, y}+p_{1}\right) .
\end{aligned}
$$

Both equations (62) and (65) satisfy the correct Euclidean limit, and each of them could be solved by power series solution. In fact, there are no polynomial solutions for real values of the quantum number $\nu$. Consequently, the eigenvalues $\nu$ can take on any positive value, and the spectrum for the energy is continuous, as in the Euclidean case. In the hypergeometric case, both functions $Y(y)$ and $Z\left(z_{x}\right)$ turn out to be non-normalizable functions, so the hyperbolic case can be thought of as more similar to the Euclidean case, rather than the spherical $\kappa>0$ case.

\section{References}

[1] Abraham R. and Marsden J., Foundations of Mechanics, $2^{\text {nd }}$ Edn, BenjaminCummings, Reading 1978.

[2] Bracken P., Motion on Constant Curvature Spaces and Quantization Using Noether Symmetries, Chaos 24 (2014) 043128.

[3] Bracken P., Quantization of a Particle on a Two-Dimensional Manifold of Constant Curvature, J. Math. Phys. 55 (2014) 102102.

[4] Cariñena J., Rañada M. and Santander M., The Quantum Free Particle on Spherical and Hyperbolic Spaces, J. Math. Phys. 52 (2011) 072104. 
[5] Cariñena J., Rañada M. and Santander M., The Quantum Harmonic Oscillator on the Sphere and the Hyperbolic Plane: $\kappa$-Dependent Formalism, Polar Coordinates and Hypergeometric Functions, J. Math. Phys. 48 (2007) 102106.

[6] Cariñena J., Rañada M. and Santander M., A Quantum Exactly Solvable NonLinear Oscillator with Quasi-Harmonic Behavior, Ann. Phys. 322 (2007) 434-459.

[7] Cariñena J., Rañada M., Santander M. and Senthilvelan M., A Non-Linear Oscillator with Quasi-Harmonic Behaviour: Two- and n-Dimensional Oscillators, Nonlinearity 17 (2004) 1941-1963.

[8] Char B., Geddes K., Gonnet G., Leong B., Monagen M. and Watt S., Maple V Library Manual, Springer, New York 1994.

[9] Corben H. and Stehle P., Classical Mechanics, $2^{\text {nd }}$ Edn, Dover, New York 1994.

[10] da Costa R. C. T., Quantum Mechanics of a Constrained Particle, Phys. Rev. A 23 (1981) 1982-1987.

[11] Lakshmanan M. and Rajackar S., Nonlinear Dynamics, Integrability, Chaos and Patterns, Springer, Berlin 2003.

[12] Matsutani S., On the Essential Algebraic Aspects of Submanifold Quantum Mechanics, J. Geom. Symmetry Phys. 2 (2004) 18-26.

[13] Mladenov I., Quantization on Curved Manifolds, Geometry Integrability \& Quantization 3 (2001) 64-104.

[14] McKenna I. and Wan K., The Role of the Connection in Geometric Quantization, J. Math. Phys. 25 (1984) 1798-1803.

[15] Sniatycki J., Geometric Quantization and Quantum Mechanics, Springer, New York 1980.

[16] Xun D. M., Liu Q. H. and Zhu X. M., Quantum Motion on a Torus as a Submanifold Problem in a Generalized Dirac's Theory of Second Class Constraints, Ann. Phys. 338 (2013) 123-133.

Paul Bracken

Department of Mathematics

University of Texas

Edinburg, TX 78540

USA

E-mail address: bracken@utpa . edu 